\title{
Integrative genomic, proteomic and phenotypic studies of Leishmania donovani strains revealed genetic features associated with virulence and antimony-resistance
}

\author{
Zhiwan Zheng ${ }^{1}$, Jianping Chen ${ }^{1,2}$, Guangxu Ma ${ }^{3,4}$, Abhay R. Satoskar ${ }^{5,6}$ and Jiao Li ${ }^{{ }^{*}}$
}

\begin{abstract}
Background: Leishmaniasis is a neglected tropical disease affecting millions of people worldwide. Emerging drug resistance of Leishmania species poses threaten to the effective control and elimination programme of this neglected tropical disease.

Methods: In this work, we conducted drug-resistance testing, whole genome resequencing and proteome profiling for a recently reported clinical isolate with supposed drug resistance (HCZ), and two reference sensitive strains (DD8 and 9044) of Leishmania donovani, to explore molecular mechanisms underlying drug resistance in this parasite.

Results: With reference to DD8 and 9044 strains, HCZ isolate showed higher-level virulence and clear resistance to antimonials in promastigote culture, infected macrophages and animal experiment. Pairwise genomic comparisons revealed genetic variations (86 copy number variations, 271 frameshift mutations in protein-coding genes and two site mutations in non-coding genes) in HCZ isolate that were absent from the reference sensitive strains. Proteomic analysis indicated different protein expression between HCZ isolate and reference strains, including 69 exclusively detected proteins and 82 consistently down-/upregulated molecules in the HCZ isolate. Integrative analysis showed linkage of 12 genomic variations (gene duplication, insertion and deletion) and their protein expression changes in $\mathrm{HCZ}$ isolate, which might be associated with pathogenic and antimony-resistant phenotype. Functional annotation analyses further indicated that molecules involved in nucleotide-binding, fatty acid metabolism, oxidation-reduction and transport might play a role in host-parasite interaction and drug-resistance.
\end{abstract}

Conclusions: This comprehensive integrative work provided novel insights into the genetic basis underlying virulence and resistance, suggesting new aspects to be investigated for a better intervention against L. donovani and associated diseases.

Keywords: Leishmania donovani, Antimony resistance, Whole genome resequencing, Proteome profiling, Genetic variations

*Correspondence: joyleeql2019@163.com

1 Department of Pathogenic Biology, West China School of Basic Medical Sciences and Forensic Medicine, Sichuan University, Chengdu, China

Full list of author information is available at the end of the article

\section{Background}

Leishmaniasis, a neglected tropical disease, is caused by the infection with Leishmania species, affecting millions of people in approximately 100 endemic countries $[1,2]$. This parasitic disease is transmitted by the bite of phlebotomine sand flies, and have three main forms: cutaneous, mucocutaneous and visceral leishmaniasis (VL)

c) The Author(s) 2020. This article is licensed under a Creative Commons Attribution 4.0 International License, which permits use, sharing, adaptation, distribution and reproduction in any medium or format, as long as you give appropriate credit to the original author(s) and the source, provide a link to the Creative Commons licence, and indicate if changes were made. The images or other third party material in this article are included in the article's Creative Commons licence, unless indicated otherwise in a credit line to the material. If material is not included in the article's Creative Commons licence and your intended use is not permitted by statutory regulation or exceeds the permitted use, you will need to obtain permission directly from the copyright holder. To view a copy of this licence, visit http://creativecommons.org/licenses/by/4.0/. The Creative Commons Public Domain Dedication waiver (http://creativecommons.org/publicdomain/zero/1.0/) applies to the data made available in this article, unless otherwise stated in a credit line to the data. 
[3]. Although most people have a silent infection, some advanced cases of cutaneous leishmaniasis can cause ulcers, and VL is almost always fatal without proper treatment, especially the advanced one $[3,4]$. In the past decades, the intensive vector control and a better access to diagnosis and treatment facilities have successfully and substantially reduced the number of VL in Asia [2, 5-7]. However, leishmaniasis remains to be one of the povertyrelated diseases in underprivileged countries and regions $[1,2,8]$. No vaccines are currently available for clinical use to prevent the infection and reinfection by Leishmania species, although progress has been made in this field [9-11]. Chemotherapy is still the first-line option for the treatment and elimination of Leishmania infection and related diseases $[12,13]$.

Drug resistance of Leishmania species usually results in treatment failure and relapse of leishmaniasis, posing a considerable threaten to the effective control of this disease [14]. Although liposomal amphotericin B, miltefosine and pentavalent antimonials have been widely used for the treatment of VL in the past decades, an increase of treatment failure and sequela of infection has been frequently reported in recent years, particularly in endemic countries and regions (e.g. Nepal and Southeast Asia region) [15-18]. Unfortunately, molecular mechanisms underlying the establishment of drug resistance have not yet been clearly elucidated [19], which significantly hinders the discovery of novel intervention strategies (e.g. new drugs or vaccines), and challenges the elimination of leishmaniasis by 2020 in endemic areas. Tackling these issues and challenges requires a better understanding of the molecular basis underlying drug resistance in Leishmania species.

In the past 30 years, progress has been made in unravelling the infection, genetics and evolution of Leishmania spp. at the molecular level [20-22]. Decoding the genomes of Leishmania species marked an important turning point for the molecular exploration of these pathogens [23-25]. Particularly, recent genomic and transcriptomic studies of Leishmania species significantly advanced our understanding of the genetic plasticity and molecular differences between species/strains, which might be associated with the resistance to antileishmanial drugs and treatment failure [22, 26-29]. However, it is still challenging to integrate and interpret the genetic features revealed at DNA and RNA levels to elucidate the genetic basis for drug resistance in protozoan parasites. Clearly, there is a strong need to explore the genetic plasticity and biological variations of Leishmania species at genomic and post-genomic (transcriptomic, proteomic and metabolomic) levels [30, 31].

In this study, we conducted antimony-resistant testing, genomic sequencing and proteomic profiling of a clinical isolate (HCZ) and two drug-susceptible (DD8 and 9044) strains of $L$. donovani. Comparative studies were performed to identify genetic variations and specificities at DNA and protein levels between the resistant and susceptible phenotypes. Molecular mechanisms underlying virulence and resistance in $L$. donovani were explored by comparing and integrating the phenotypic features, genomic variations and proteomic differences among HCZ isolate, DD8 and 9044 strains.

\section{Methods}

Procurement of parasites, drugs, cell and animals

A Leishmania clinical isolate (MHOM/CN/2016/ SCHCZ) was obtained from bone marrow puncture fluid of a VL patient at West China Hospital, Sichuan University, China. The patient was treated with 4 courses of antimonials treatment and then relapsed. The bone marrow puncture fluid was obtained after the relapse, then inoculated with three laboratory golden hamsters by intraperitoneal injection, $0.3 \mathrm{ml}$ per hamster. Clinical isolate (MHOM/CN/2016/SCHCZ) and reference sensitive strains (MHOM/CN/IN/80/DD8 and $\mathrm{MHOH} /$ $\mathrm{CN} / 90 / 9044)$ of $L$. donovani have been preserved and passaged in the golden hamsters. Livers and spleens of sick golden hamsters were homogenized and maintained in M199 medium (Sigma-Aldrich St. Louis, MO, USA) supplemented with $10 \%$ fetal bovine serum (FBS; Gibco, Franklin, TN, USA) and antibiotics $(100 \mathrm{U} / \mathrm{ml}$ penicillin and $100 \mu \mathrm{g} / \mathrm{ml}$ streptomycin; Hyclone, Logan, UT, USA) at $\mathrm{pH} 7.4$ and $28^{\circ} \mathrm{C}$ for $5-7$ days to culture promastigotes.

Sodium stibogluconate (SSG; MCE, Monmouth Junction, NJ, USA) and potassium antimonyl tartrate trihydrate (PAT; Sigma-Aldrich St. Louis, MO, USA) were dissolved in ultrapure water for a stock concentration of $10 \mathrm{mM}$. A murine macrophage stable cell line RAW 264.7 (Jennio, Guangzhou, China) was recovered and maintained according to the manufacturer's instructions. Golden hamsters (Mesocricetus auratus, 8-10 weeksold, female) were purchased from the Chengdu Institute of Biological Products Co., Ltd, Chengdu, China. Female wild-type BALB/c mice aged 6-7 weeks were purchased from Dassy (Experimental animals Co., Ltd, Chengdu, China), and maintained under standard conditions.

\section{In vitro promastigote culture}

To test the likely antimony-resistance of the clinical isolate (HCZ) of $L$. donovani [18], logarithmic phase promastigotes of $\mathrm{HCZ}$ isolate and reference sensitive strains DD8 and 9044 were harvested, washed, and cultured in M199 medium (Sigma-Aldrich) supplemented with 10\% FBS (Gibco), antibiotics (100 U/ml penicillin and $100 \mu \mathrm{g} / \mathrm{ml}$ streptomycin) and $65 \mu \mathrm{M}$ of PAT (cf. [32]), at a cell density of $1.0 \times 10^{6}$ cells/well. Same volume of 
phosphate-buffered saline (PBS) was used as control. Following 24 and $48 \mathrm{~h}$ of incubation with PAT, the survival rate of promastigotes of $L$. donovani strains tested was determined by a flow cytometry analysis. In brief, promastigote samples of $\mathrm{HCZ}$ isolate, DD8 and 9044 strains were collected by centrifuge at $800 \times g$ and purified by three washes in $\mathrm{PBS}$ at $4{ }^{\circ} \mathrm{C}$; purified promastigotes were then processed using a FITC Annexin V Apoptosis Detection Kit I (BD, Franklin Lakes, NJ, USA) according to the manufacturer's instructions, then analysed by a flow cytometry machine (CytoFLEX LX; BD). Inhibition rate $(\%)=100 \%-[$ (No. of live promastigotes in treated sample/ No. of live promastigotes in control) $\times 100 \%]$.

\section{Macrophage infection}

RAW 264.7 cells $\left(\sim 5.0 \times 10^{5}\right.$ cells/per well $)$ were plated on round glass coverslips in 24-well plates and allowed to adhere to the slides for $24 \mathrm{~h}$ at $37{ }^{\circ} \mathrm{C}, 5 \% \mathrm{CO}_{2}$ with RPMI-1640 medium (Sigma-Aldrich) supplemented with 10\% FBS (Gibco). Stationary phase promastigotes $\left(\sim 5.0 \times 10^{6}\right.$ cells/per well $)$ of HCZ isolate, DD8 and 9044 strains were added into wells, and incubated at 37 ${ }^{\circ} \mathrm{C}, 5 \% \mathrm{CO}_{2}$ for $6 \mathrm{~h}$. Next, the non-infected promastigotes were removed, and the infected macrophages were incubated at $37{ }^{\circ} \mathrm{C}$ in $5 \% \mathrm{CO}_{2}$ with fresh medium without drugs for $24 \mathrm{~h}$. The medium was then removed, and fresh culture medium supplemented with $10 \mu \mathrm{M}$ SSG (cf. [33]) were added into wells for 2 days, with PBS used as control. At 24 and $48 \mathrm{~h}$, the glass coverslips were fixed in methanol (Solarbio, Beijing, China) and stained with Wright's stain (Solarbio) to calculate the number of intracellular amastigotes under light microscopy by counting 200 macrophages per slide. Inhibition rate (\%) $=100 \%-[($ No. of amastigotes in treated sample/ No. of amastigotes in control) $\times 100 \%]$.

\section{Animal infection and treatment with SSG}

All experimental mice under a standard condition were randomly divided into three groups, with 12 mice included in each group. Stationary phase promastigotes, collected from the culture medium, were centrifuged at $1000 \times g$ for $10 \mathrm{~min}$, resuspend in PBS. Using an intravenous approach, promastigotes $\left(\sim 1.0 \times 10^{7}\right.$ cells $)$ of HCZ isolate, DD8 and 9044 strains of $L$. donovani were inoculated in three groups of mice, respectively. BALB/c mice of three groups were treated with SSG at a single dose of $70 \mathrm{mg} / \mathrm{kg}$ (cf. [34]) body weight of the animal or PBS via intraperitoneal injection at 14 days after infection. All mice were euthanized and dissected after 14 days of treatment. The livers and spleens were harvested, weighed, and smears (Wright's staining) were prepared and examined by light microscopy. The parasite burdens were indicated as Leishman-Donovan Units $($ LDU $)=$ No. of amastigotes per 1000 nucleated cells $\times$ liver or spleen weight $(\mathrm{g})$.

\section{DNA extraction and sequencing}

Genomic DNA were isolated from promastigotes representing each of the $\mathrm{HCZ}$ isolate, DD8 and 9044 strains of L. donovani using TIANamp Genomic DNA Kit (Tiangen, Beijing, China) according to the manufacturer's instructions. In brief, purified stationary phase promastigotes $\left(\sim 4 \times 10^{8}\right.$ cells $)$ were transferred to a sterile microcentrifuge tube containing lysis buffer, from which the released genomic DNA was bound to column, washed with washing buffer, and then eluted with nuclease-free water. DNA samples representing the $\mathrm{HCZ}$ isolate, DD8 and 9044 strains were stored at $-20^{\circ} \mathrm{C}$ until use.

The quality and intensity of DNA samples were measured using a Qubit Fluorometer (Thermo Fisher Scientific, Rochester, NY, USA). Sequencing libraries (pair-end) representing each $L$. donovani samples ( $\mathrm{HCZ}$ isoalte, DD8 and 9044 strains) were constructed using an internal DNA 200 bp-800 bp Insert Size Pooling Library Construction Kit (BGI, Shengzheng, China). In brief, $1 \mu \mathrm{g}$ genomic DNA was randomly fragmented, incubated with End Repair Mix, combined with A-Tailing Mix, ligated to Illumina adapters, amplified with PCR Primer Cocktail and PCR Master Mix, and validated by the Agilent 2100 Bioanalyzer and ABI StepOnePlus Real-Time PCR System. The qualified libraries were sequenced on Illumina Hiseq4000 (PE150) platform.

\section{Genomic mutation analysis}

Pair-end raw reads were processed to remove sequencing adaptors and low-quality reads for quality control. Processed reads were mapped to the reference genome of $L$. donovani (BPK282A1 strain; BioProject: PRJEA61817) using the BWA software v.0.7.15 [35]. Following genome mapping, contaminated reads were removed while mapped reads were summarised for statistical analyses of mapping rate, sequencing depth and genomic coverage for HCZ isolate, DD8 and 9044 strains of each $L$. donovani strain. Structural variation (SV), copy number variation $(\mathrm{CNV})$, single nucleotide polymorphism (SNP) and insertion/deletion (indel) were detected by comparing mapped reads with the reference genome using BreakDancer v.1.1 [36], CNVnator v.0.3 [37] and GATK v.4.0 [38]. Detected SNPs were filtered using parameters ExcessHet $>54.69$, QD $<2.0$, MQ $<40.0$, FS $>60.0$, SOR $>$ 3.0, MQRankSum < -12.5 and ReadPosRankSum < -8.0. Identified Indels were checked based on thresholds ExcessHet $>54.69$, QD $<2.0$, FS $>200.0$, SOR $>10.0$, MQRankSum $<-12.5$ and ReadPosRankSum $<-8.0$. Filtered SNPs and Indels were annotated using ANNOVAR software [39]. 


\section{Protein isolation and LC-MS/MS}

Proteins were extracted from the same batch of $L$. donovani samples used for the genomic DNA isolation. In brief, the purified promastigotes were incubated in lysis buffer ( $8 \mathrm{M}$ urea and $1 \%$ protease inhibitor cocktail) at 4 ${ }^{\circ} \mathrm{C}$ for $3 \mathrm{~min}$, sonicated on ice for three times using a high intensity ultrasonic processor (Scientz, Ningbo, China). Sonicated samples were centrifuged at $12,000 \times g$ at $4^{\circ} \mathrm{C}$ for $10 \mathrm{~min}$ to remove debris. Supernatant was collected, and the protein concentration was measured using a BCA Protein Assay Kit (Thermo Fisher Scientific).

For each of the L. donovani samples ( $\mathrm{HCZ}$ isolate, DD8 and 9044 strains), proteins were subjected to an insolution reduction, alkylation and digestion approach. In brief, $300 \mu \mathrm{g}$ protein aliquots $(n=3)$ was reduced with 5 $\mathrm{mM}$ dithiothreitol at $56{ }^{\circ} \mathrm{C}$ for $30 \mathrm{~min}$, alkylated with 11 $\mathrm{mM}$ iodoacetamide at room temperature in darkness for $15 \mathrm{~min}$, digested with trypsin (1:50 trypsin-to-protein) at $37^{\circ} \mathrm{C}$ overnight, and further digested with trypsin (1:100 trypsin-to-protein) at $37^{\circ} \mathrm{C}$ for $4 \mathrm{~h}$. The processed samples were dissolved in $1.0 \%(\mathrm{v} / \mathrm{v})$ formic acid, and then subjected to liquid chromatography-mass spectrometry/mass spectrometry (LC-MS/MS) analysis using a Q ExactiveTM Plus Orbitrap mass spectrometer (Thermo Fisher Scientific) coupled online to the EASY-nLC 1000 UPLC system.

\section{Proteomic data analysis}

Mass spectrometry data were processed using MaxQuant search engine v.1.5.2.8 [40]. In brief, tandem mass spectra were search against the reference proteome of $L$. donovani (BPK282A1 strain; BioProject: PRJEA61817) to identify and quantify peptides. Trypsin/P was specified as cleavage enzyme ( $\leq 4$ missing cleavages), mass tolerances for precursor ions were set as $20 \mathrm{ppm}$ for the first search and $5 \mathrm{ppm}$ for the main search, and mass tolerance for fragment ions as $0.02 \mathrm{Da}$. False discovery rate (FDR) was adjusted to < $1 \%$ and minimum score for modified peptides was set $>40$. Gene Ontology (GO), domain architecture, Kyoto Encyclopedia of Genes and Genomes (KEGG) and subcellular localisation annotations were performed for the identified proteins based on UniProt-GOA database, InterPro, KEGG database and Wolfpsort databases using an internal R script.

\section{Comparative and integrative analyses}

Pairwise comparisons were conducted among HCZ isolate, DD8 and 9044 strains of L. donovani at genomic and proteomic levels. Genomic mutations (e.g. CNV, SNV and frameshift for protein-coding genes) and protein abundances (i.e. qualitative and quantitative data sets) were compared between $\mathrm{HCZ}$ and DD8, $\mathrm{HCZ}$ and 9044, DD8 and 9044 to infer specific genomic mutations and protein expression patterns in HCZ isolate of $L$. donovani. Perseus software [41, 42] was used to analyse and visualise proteomic datasets. Principal components analysis (PCA) and hierarchical cluster analysis (HCA) were performed to analyse differences among the proteomic datasets representing distinct strains of $L$. donovani. Venn diagram was drawn using a R package Venn Diagram v.1.6.20. Cross-comparison of genomic and proteomic data was performed for an integrative analysis of the relationship between mutations at DNA level and expression changes at protein level.

\section{Statistical analysis}

All experiments were repeated a minimum of three separate times. All data analyses and graphs were performed with GraphPad Prism 6.0 software (San Diego, CA, USA). The data were analyzed using Student's t-test for comparison of two groups and were expressed as the means \pm standard deviation (SD). Significant differences were determined and designated with asterisks as follows: ${ }^{*} P<$ $0.05{ }^{* * *} P<0.01 ;{ }^{* * *} P<0.001$ and ${ }^{* * * *} P<0.0001$.

\section{Results}

Leishmania donovani $\mathrm{HCZ}$ isolate was antimony-resistant in promastigote culture and in infected macrophages in vitro

Following incubation with PAT in vitro, flow cytometry analysis revealed similar survival rates of promastigotes between DD8 and 9044 strains, but different survival rates between $\mathrm{HCZ}$ isolate and DD8 strain, and between HCZ isolate and 9044 strain of L. donovani. Specifically, compared with the low survival rates of DD8 (38.61\% and $16.72 \%)$ and 9044 strains (39.83\% and $18.42 \%)$ of $L$. donovani after 24 and $48 \mathrm{~h}$ of incubation with PAT (65 $\mu \mathrm{M}), \mathrm{HCZ}$ isolate appeared to be more robust $(82.04 \%$ and $79.92 \%$ ) (Fig. 1a). In addition, lower number of intracellular amastigotes were observed in the macrophages infected with 9044 and DD8 strains after treatment (SSG, $10 \mu \mathrm{M}$ ), whereas more amastigotes were found in $\mathrm{HCZ}$ isolate infected macrophages (Fig. 2a). For HCZ isolate, the inhibitory effects of PAT on promastigotes ( $\mathrm{HCZ} v \mathrm{~s}$ DD8: $t_{(8)}=10.0549, P<0.0001,24 \mathrm{~h} ; t_{(8)}=13.9502, P<$ 0.0001, 48 h) (HCZ vs 9044: $t_{(8)}=11.0015, P<0.0001,24$ $\left.\mathrm{h} ; t_{(8)}=15.7577, P<0.0001,48 \mathrm{~h}\right)$ in vitro and SSG on intracellular amastigotes in macrophages (HCZ vs DD8: $t_{(8)}=8.4122, P<0.0001,24 \mathrm{~h} ; t_{(8)}=12.105, P<0.0001$, $48 \mathrm{~h})\left(\mathrm{HCZ}\right.$ vs 9044: $t_{(8)}=6.5992, P=0.0002,24 \mathrm{~h} ; t_{(8)}=$ 10.7526, $P<0.0001,48 \mathrm{~h}$ ) were significantly weaker than 


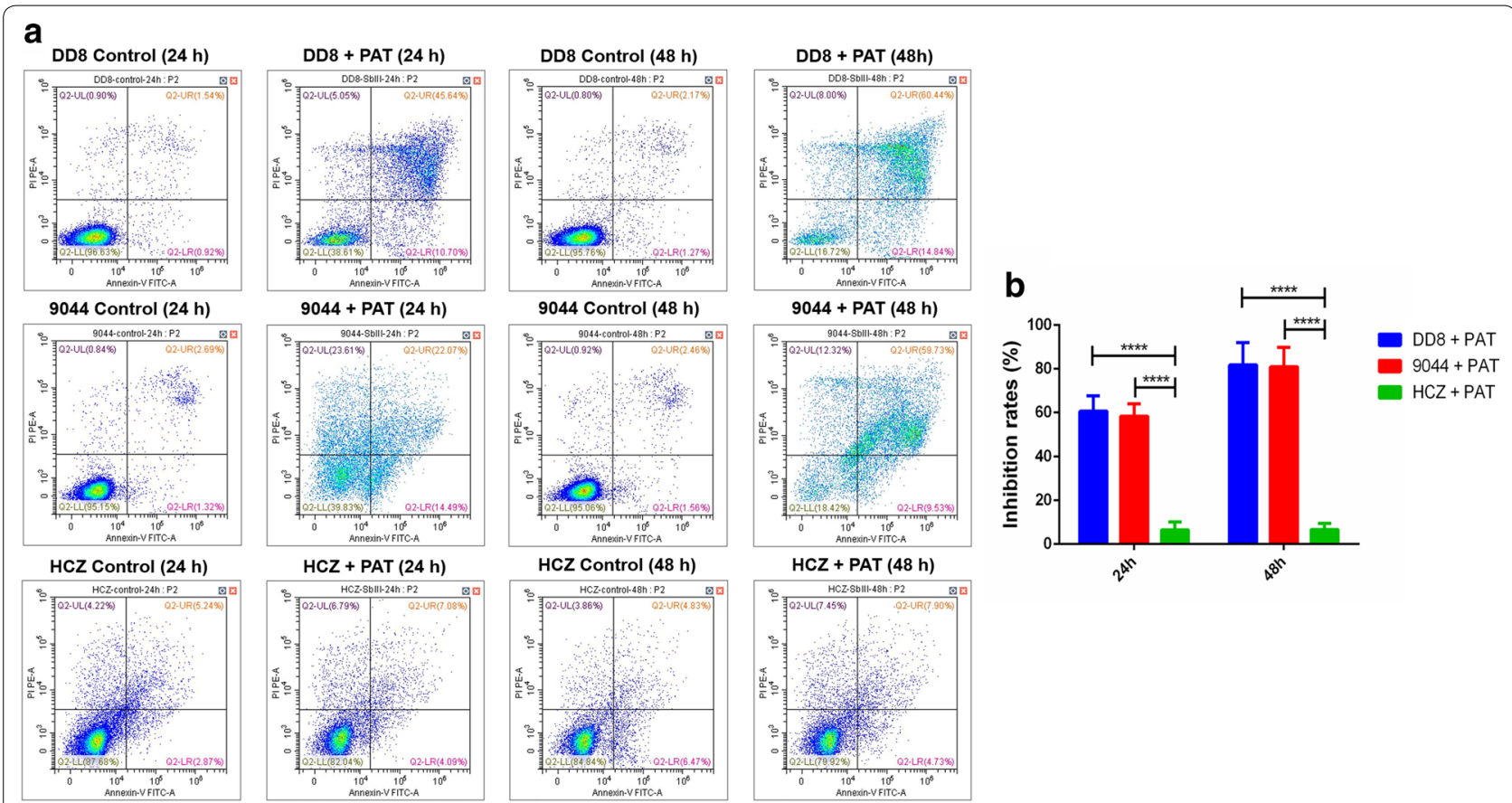

Fig. 1 Flow cytometry analysis of Leishmania donovani promastigotes exposed to PAT and inhibition rates of PAT on promastigotes. a Survival rates of promastigotes representing reference sensitive strains (9044 and DD8), and clinical isolate (HCZ) of L. donovani after 24 and 48 h of incubation with PBS (control) and PAT are indicated in the lower left quadrant (LL quadrant). $\mathbf{b}$ Inhibition rates of PAT on the survival of promastigotes of DD8 and 9044 strains and HCZ isolate after 24 and $48 \mathrm{~h}$ of incubation. All experiments were carried out in triplicates and figures shown are representative of these experiments. ${ }^{* * *} P<0.0001$

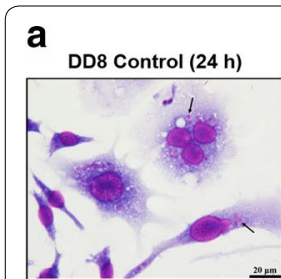

9044 Control (24 h)

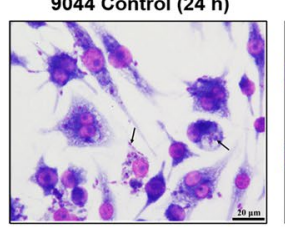

HCZ Control (24 h)

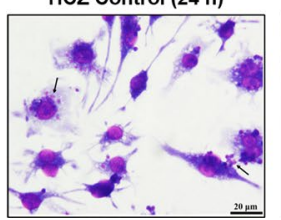

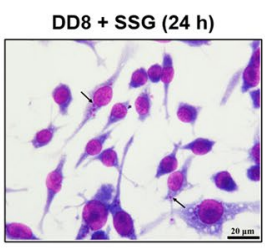

9044 + SSG (24 h)

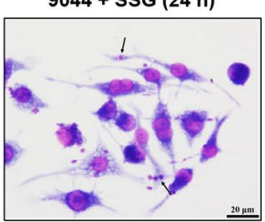

$\mathrm{HCZ}+$ SSG (24 h)

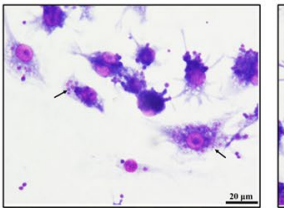

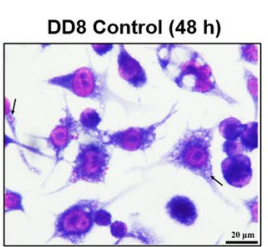

9044 Control (48 h)

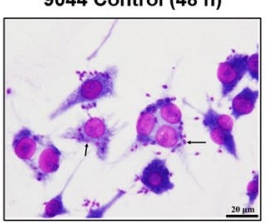

HCZ Control (48 h)

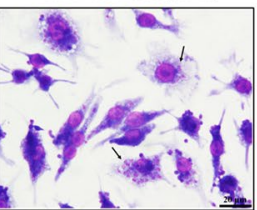

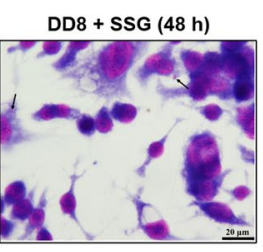

9044 + SSG (48 h)

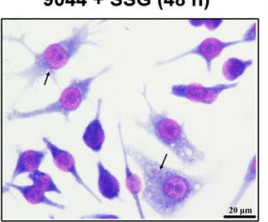

HCZ + SSG (48 h)

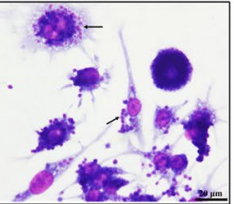

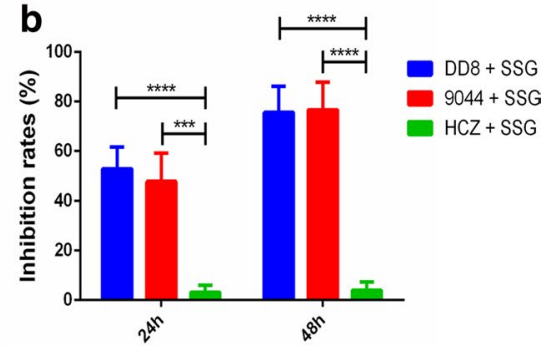

Fig. 2 Wright's staining of intracellular amastigotes in cells after treated with SSG and inhibition rates of SSG on intracellular amastigotes. a Intracellular amastigotes representing reference sensitive 9044 and DD8 strains, and HCZ isolate of L. donovani after 24 and 48 h of incubation with PBS (control) and SSG. $\mathbf{b}$ Inhibition rates of SSG on the number of intracellular amastigotes in macrophages after 24 and 48 h of treatment. At least three independent experiments were carried out and figures shown are representative of these experiments. The black arrows indicated intracellular amastigotes. ${ }^{* *} P<0.001,{ }^{* * *} P<0.0001$. Scale-bars: $20 \mu \mathrm{m}$ 
that for 9044 and DD8 strains (Figs. 1b and 2b), indicating antimony-resistance of $L$. donovani $\mathrm{HCZ}$ strain in vitro.

\section{Leishmania donovani $\mathrm{HCZ}$ isolate showed strong virulence and resistance in vivo}

To further confirm the antimony-resistant phenotype of $\mathrm{HCZ}$ isolate of $L$. donovani, resistance testing was conducted in a mice infection model. By comparing the parasite loads of livers and spleens of $\mathrm{BABL} / \mathrm{c}$ mice in SSG-treated and PBS-treated groups, HCZ isolate appeared to be more pathogenic and more resistant to antimony than DD8 and 9044 strains in vivo (Fig. 3).

Specifically, although parasite loads were observed in all mice infected with DD8 and 9044 strains, and HCZ isolate of $L$. donovani, the LDU of liver in the latter group was significantly higher than other two groups $\left(\mathrm{HCZ}+\mathrm{PBS} v s\right.$ DD8 + PBS: $t_{(4)}=4.5104, P=0.0107$; HCZ + PBS vs $9044+$ PBS: $\left.t_{(4)}=3.838, P=0.0185\right)$ (Fig. 3a). Similar to the LDU of liver, the LDU of spleen in $\mathrm{HCZ}+\mathrm{PBS}$ group was significantly higher than that in other two groups (HCZ + PBS vs DD8 + PBS: $t_{(4)}=3.6541, P=0.0217 ; \mathrm{HCZ}+\mathrm{PBS} v s 9044+\mathrm{PBS}:$ $t_{(4)}=3.4466, P=0.0261$ ) (Fig. 3b). Additionally, SSG treatment efficiently eliminated the intracellular amastigotes of 9044 and DD8 strains, but not HCZ isolate.
Specifically, the LDU of liver (DD8 + SSG vs DD8 + PBS: $t_{(4)}=4.4701, P=0.0111 ; 9044+$ SSG $v s 9044+$ PBS: $\left.t_{(4)}=5.3143, P=0.006\right)$ and spleen (DD8 + SSG $v s$ DD8 + PBS: $t_{(4)}=3.5172, P=0.0245 ; 9044+$ SSG $v s$ $9044+$ PBS: $\left.t_{(4)}=3.496, P=0.025\right)$ in DD8 and 9044 groups significantly decreased. On the contrary, the LDU of liver ( $\mathrm{HCZ}+\mathrm{SSG} v s \mathrm{HCZ}+\mathrm{PBS}: t_{(4)}=0.6506$, $P=0.5508)$ and spleen $\left(\mathrm{HCZ}+\mathrm{SSG} v s \mathrm{HCZ}+\mathrm{PBS}: t_{(4)}\right.$ $=0.3546, P=0.7408)$ in $\mathrm{HCZ}$ infected $\mathrm{BABL} / \mathrm{c}$ mice did not significantly reduce after treatment of SSG, showing clear antimony-resistance of $L$. donovani $\mathrm{HCZ}$ isolate in vivo (Fig. 3).

\section{Genomic mutations identified in L. donovani $\mathrm{HCZ}$ isolate, 9044 and DD8 strains}

More than 7.80 million raw reads were produced for each L. donovani isolate or strain (HCZ, 9044 and DD8) (Additional file 1: Table S1). By aligning the raw reads to the reference genome of this parasite (BPK282A1 strain; BioProject: PRJEA61817), at least 4.79 million reads were mapped for each of the three $L$. donovani strains (Table 1). Specifically, mapped reads for the drugresistant clinical isolate $(\mathrm{HCZ})$ of $L$. donovani covered $>90 \%$ of the reference genome (BPK282A1 strain) at a $\geq 10$-fold coverage (Table 1). Similar genome coverages $(94.79 \%$ and $94.66 \%)$ were also achieved for the
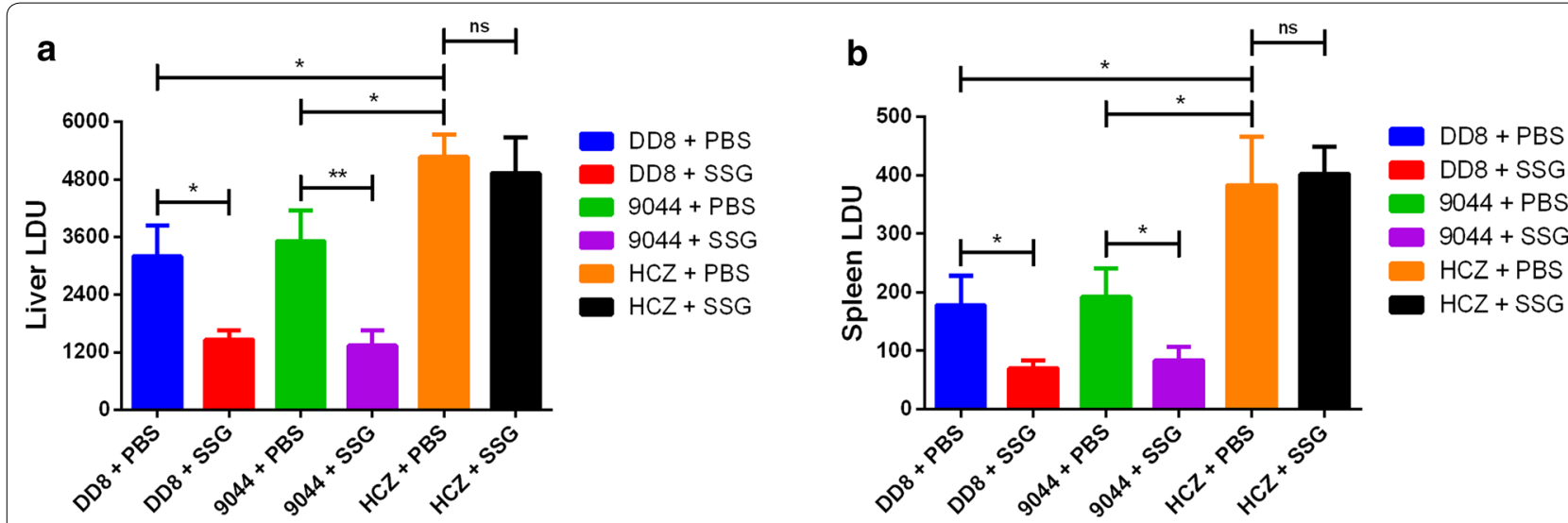

Fig. 3 Parasite loads during Leishmania donovani infection after treatment with SSG or PBS. Liver (a) and spleen (b) parasite loads in SSG- or PBS-treated L. donovani (DD8, 9044 and HCZ strains) infected BALB/C mice. Parasite burdens in spleen and liver were expressed as LDU \pm SD of the mean. The data are mean values from three independent experiments. ${ }^{*} P<0.05,{ }^{* *} P<0.01$, ns: no significance

Table 1 Statistical summary of genome resequencing and genomic analyses of L. donovani strains

\begin{tabular}{|c|c|c|c|c|c|c|c|}
\hline Strain & Mapped reads & Coverage $10 \times(\%)$ & CNVs & $5^{\prime}$ UTRs & Frameshifts & Stop-gain & Stop-loss \\
\hline $\mathrm{HCZ}$ & $4,791,654$ & 90.54 & 517 & 3 & 549 & 56 & 22 \\
\hline 9044 & $10,197,272$ & 94.79 & 670 & 2 & 431 & 59 & 22 \\
\hline DD8 & $10,051,566$ & 94.66 & 715 & 0 & 122 & 9 & 3 \\
\hline
\end{tabular}


drug-susceptible strains (9044 and DD8) of this parasite (Table 1).

By comparing the mapped reads with the reference genome (BPK282A1 strain), hundreds of structural variations including 517, 670 and 715 copy number variations (CNVs) were identified in $L$. donovani $\mathrm{HCZ}$ isolate, 9044 and DD8 strains studied, respectively (Table 1; Additional file 1: Table S1). Comparisons with the reference genome (BPK282A1 strain) also revealed thousands of single nucleotide polymorphisms (SNPs) and single nucleotide variations (SNV; i.e. insertions and deletions) in the genomes of the $\mathrm{HCZ}$ isolate, 9044 and DD8 strains (Additional file 1: Table S1). Specifically, compared with the reference strain, $>130,000$ SNPs were identified in $L$. donovani $\mathrm{HCZ}$ isolate, including 50,680 sites in proteincoding regions, 23 in non-coding RNA (ncRNA)-coding regions, and three in $5^{\prime}$ untranslated regions ( $5^{\prime} \mathrm{UTRs}$ ). These variations resulted in numbers of synonymous and nonsynonymous mutations in protein-coding and noncoding genes, such as frameshift $(n=549,431$ and 122), stop-gain ( $n=56,59$ and 9) and stop-loss $(n=22,22$ and 3) mutations in the L. donovani HCZ, 9044 and DD8 strains, respectively (Table 1; Additional file 1: Table S1).

\section{Specific genomic mutations identified in the resistant clinical isolate $(\mathrm{HCZ})$ of $L$. donovani}

Genetic mutations exclusively occurred in the clinical isolate of $L$. donovani might contribute to fitness-gains allowing for virulence and resistance. By pairwise comparisons (DD8 vs 9044; DD8 vs HCZ; 9044 vs HCZ), genetic variations in $\mathrm{HCZ}$ isolate but not in DD8 and
9044 strains were identified, including 86 copy number variations and 271 frameshift mutations (e.g. stop-gain and stop-loss mutations) in protein-coding genes, and two site mutations in non-coding genes (Additional file 1: Tables S2-S5). Specifically, one site mutation (adenine to cytosine) in the coding region and one insert mutation in the 5' UTR were identified for a histone $\mathrm{H} 3$ protein-coding gene homologue (locus tag: DBPK_101060) (Fig. 4a). In addition, a site mutation was detected in the coding region of a transfer RNA (LDBPK_10tRNA3) coding gene orthologue, which was inferred to change the secondary structure of this molecule (Fig. $4 \mathrm{~b}$ ).

\section{Proteomes of $L$. donovani HCZ, 9044 and DD8 strains}

To verify the genetic mutations in protein-coding genes and explore its biological influence at post-genomic level, proteomic analysis was conducted for $L$. donovani $\mathrm{HCZ}$, DD8 and 9044 strains. In total, 544,057 secondary spectrums were detected from the three strains, $86.5 \%$ of which were matched to the reference proteome database for $L$. donovani (BPK282A1 strain; BioProject: PRJEA61817) (Table 2). From these mapped spectrums, 73,792 peptides were identified, representing 7412 proteins/protein groups of $L$. donovani strains analysed (Table 2; Additional file 1: Table S6). Based on unique peptides detected ( $n=35,752,15,761$ and 36,190$), 7287$, 7267 and 6489 proteins were identified in HCZ, 9044 and DD8 strains of $L$. donovani, respectively. Most of these proteins were detected with $\geq 2$ unique peptides, and the number of proteins that could be quantified $(n=$ 6122, 5918 and 3962) varied among the three strains of

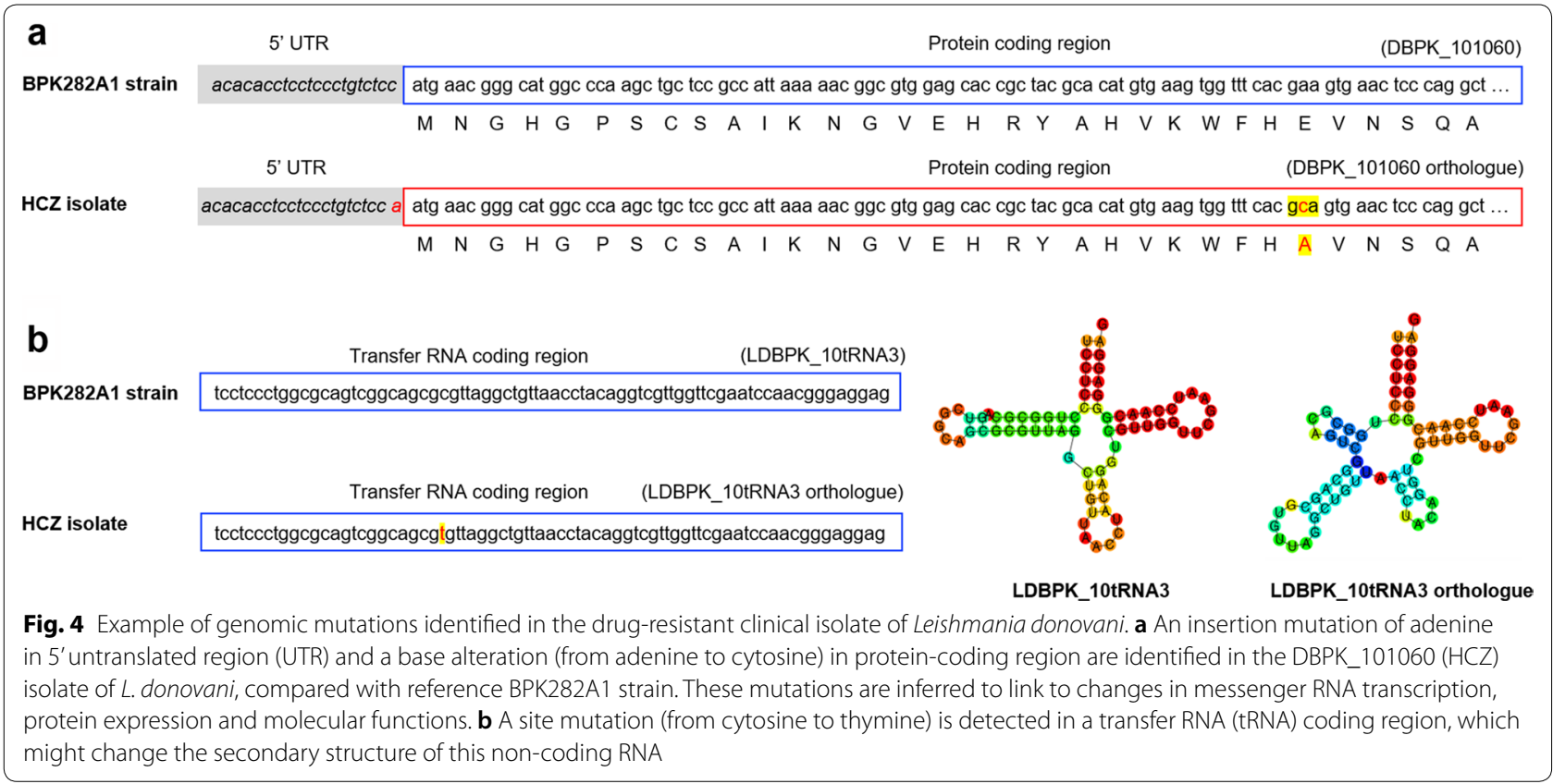


Table 2 Statistical summary of proteomic analyses of $L$. donovani strains

\begin{tabular}{|c|c|c|c|c|c|c|c|}
\hline Strain & Total spectrum & Matched spectrum (\%) & Total peptides & Total proteins & Unique peptides & $\begin{array}{l}\text { Identified } \\
\text { proteins }\end{array}$ & $\begin{array}{l}\text { Quantified } \\
\text { proteins }\end{array}$ \\
\hline $\mathrm{HCZ}$ & 544,057 & $470,565(86.5 \%)$ & 73,792 & 7412 & 35,752 & 7287 & 6122 \\
\hline 9044 & & & & & 15,761 & 7267 & 5918 \\
\hline DD8 & & & & & 36,190 & 6489 & 3962 \\
\hline
\end{tabular}

L. donovani (Table 2, Fig. 5). Although similar number of proteins were detected in the resistant $\mathrm{HCZ}$ isolate and susceptible 9044 strain (Table 2), molecules identified in the latter appeared to be expressed with lower intensities (Fig. 5), suggesting potential molecular regulations at post-genomic level.

\section{Specific protein expression changes in the resistant clinical} isolate of $L$. donovani

Different profiles of protein expression were detected among $L$. donovani strains studied in this study (Fig. 6).
A principal components analysis of proteomic data sets showed three distinct clusters representing HCZ, 9044 and DD8 strains of $L$. donovani (Fig. 6a), suggesting important biological differences between the drug-resistant and drug-susceptible strains of this parasite. Pairwise comparisons of these proteomic data sets revealed that 6400 proteins $(86.34 \%)$ were detected in all strains, and some molecules were exclusively identified in $\mathrm{HCZ}$, 9044 or DD8 strain (Fig. 6b, Additional file 1: Table S7). Specifically, 69 proteins were only detected in the resistant clinical isolate (HCZ) of $L$. donovani, 51 proteins in

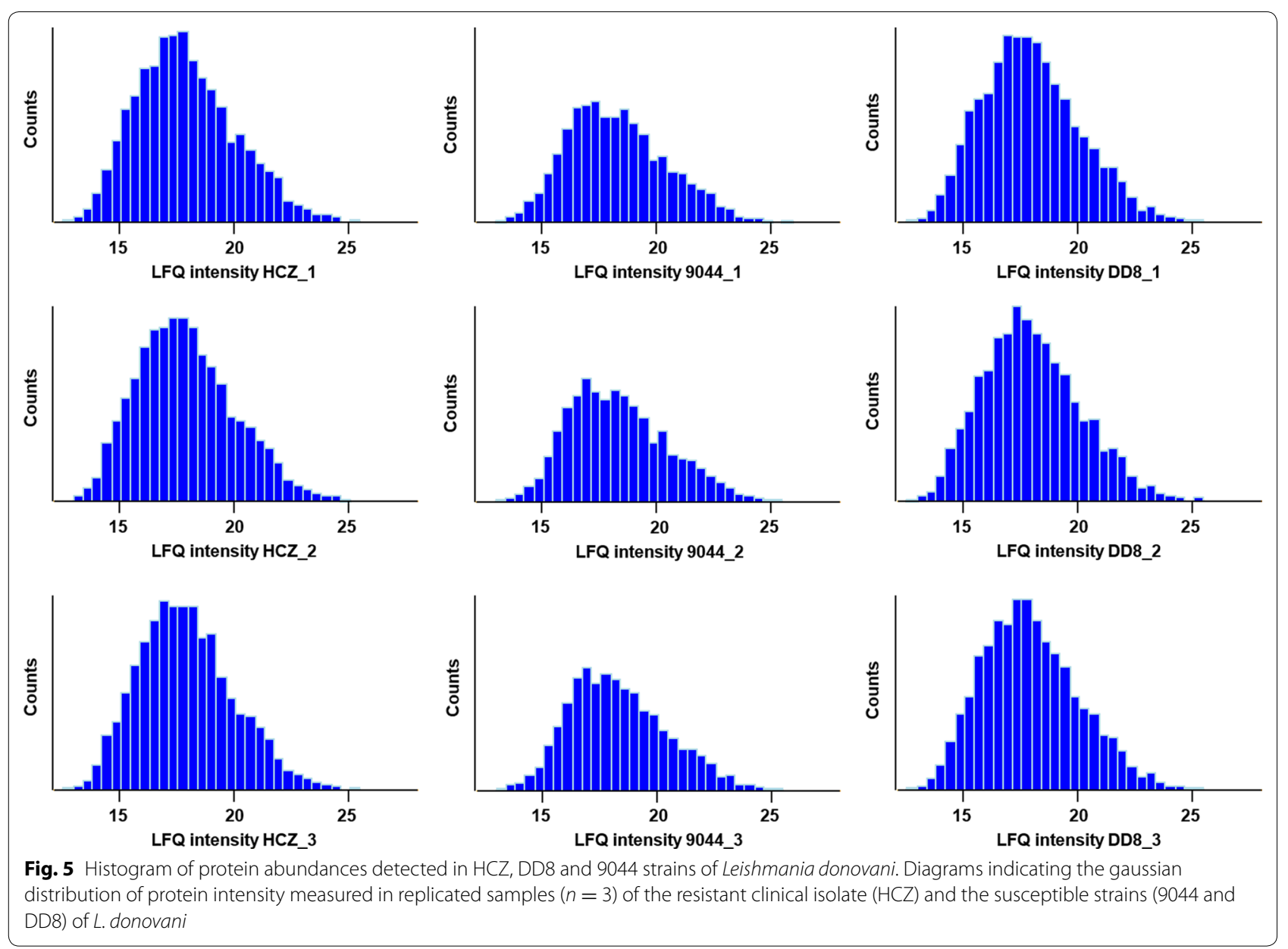



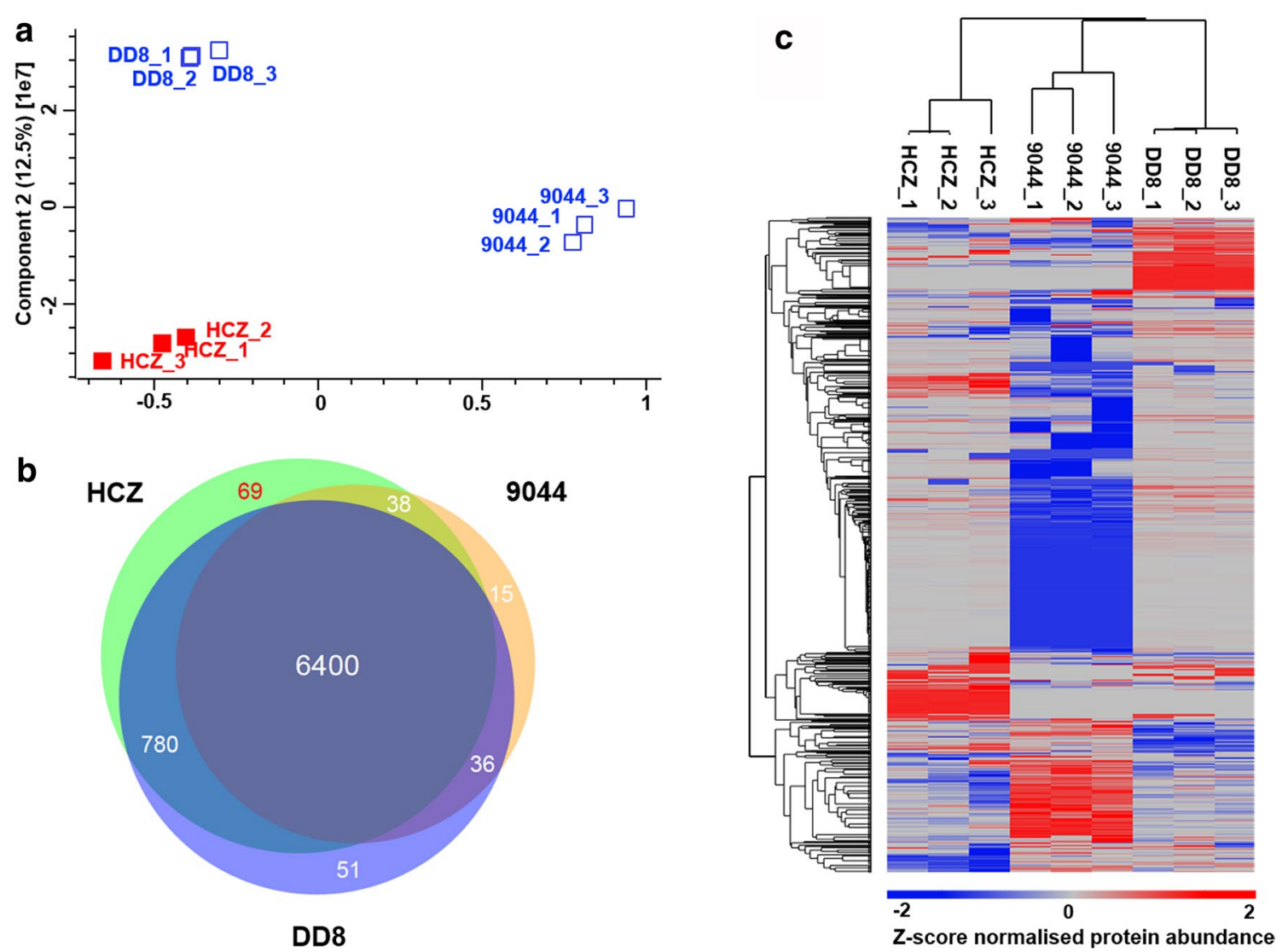

Fig. 6 Comparative analysis of protein expression among HCZ, DD8 and 9044 strains of Leishmania donovani. a Principal components analysis (PCA) of proteomic datasets of L. donovani HCZ, DD8 and 9044 strains. $\mathbf{b}$ Venn diagram showing the identification of proteins in HCZ, DD8 and 9044 strains of L. donovani. $\mathbf{c}$ Hierarchical cluster analysis (HCA) indicating the quantification of proteins in HCZ, DD8 and 9044 strains of L. donovani

DD8 strain, and 15 proteins in 9044 strain of this parasite (Fig 6b, Additional file 1: Table S7). Particularly, a hierarchical clustering of quantitative proteomic datasets unveiled different regulations of protein expression among HCZ, 9044 and DD8 strains of L. donovani (Fig. 6c). Such significant discrepancies among the three strains of $L$. donovani indicated differences in protein expression between the sensitive and resistant strains of this parasite.

Significant differences (FC $\geq 2$ and $P<0.05)$ in protein expression were identified between DD8 and 9044 ( $n=973), 9044$ and HCZ $(n=967)$, as well as DD8 and HCZ ( $n=433)$ of $L$. donovani (Fig. 7, Additional file 1 : Table S8). By removing the natural variations indicated between DD8 and 9044 strains, the number of proteins differentially expressed between resistant $\mathrm{HCZ}$ isolate and reference sensitive DD8/9044 strain reduced to 100, including 59 upregulated proteins (e.g. cysteine peptidase, paraflagellar rod component, dehydrogenases, glutathione S-transferase, inosine-adenosine-guanosinenucleosidehydrolase, NADH-ubiquinone oxidoreductase, nitrate reductase, phospholipid-transporting ATPase, tubulin beta chain, kinesin), 23 down-regulated molecules (e.g. ABC-thiol transporter, gamma-glutamylcysteine synthetase, glucosamine-fructose-6-phosphate aminotransferase, fatty acyl CoA syntetase 1 ), and 18 proteins with inconsistency (Additional file 1: Table S8).

\section{Integrated genomic mutations and protein expression changes in L. donovani $\mathrm{HCZ}$ isolate}

By integrating genomic and proteomic datasets, relationships between the genomic mutations identified in protein-coding genes and the abundances of detected proteins were established (Additional file 1: Tables S9 and S10). Predominant genomic mutations linked to significant changes in protein abundance were gene deletion, gene duplication, non-frameshift deletion and non-frameshift insertion. Specifically, gene duplication mutations resulted in exclusive protein expression (E9B9D7, E9BLQ8, E9BMP2 and E9BN45) in the drug-resistant clinical isolate (HCZ) (Fig. 8a, Additional file 1: Table S9). In addition, gene duplication, frameshift 

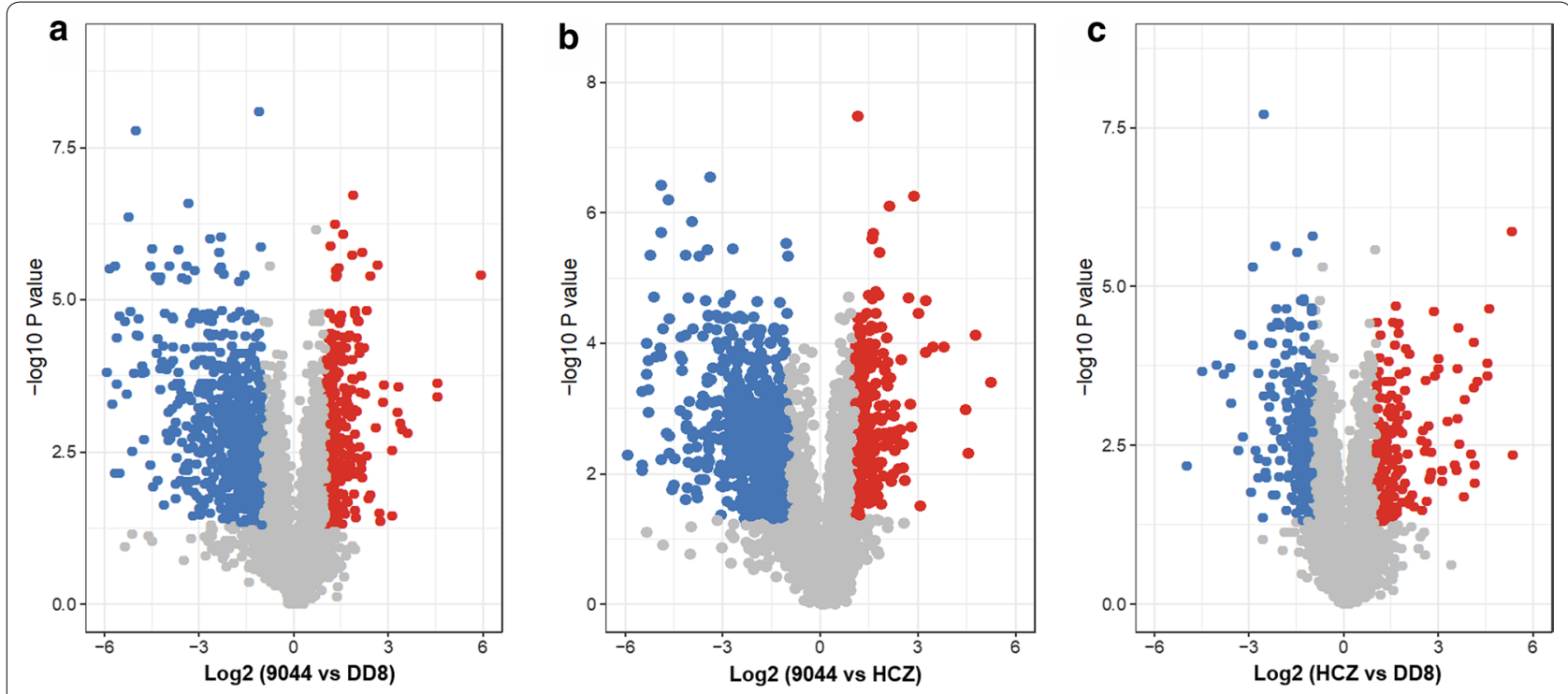

Fig. 7 Pairwise comparisons of protein expression among DD8 and 9044 strains and HCZ isolate of Leishmania donovani. Differential protein expression analyses between (a) DD8 and 9044 strains, (b) HCZ isolate and 9044 strain, and (c) HCZ isolate and DD8 strain of L. donovani. Proteins detected with significantly higher, lower and unchanged abundances in each comparison group are indicated in red, blue and grey, respectively

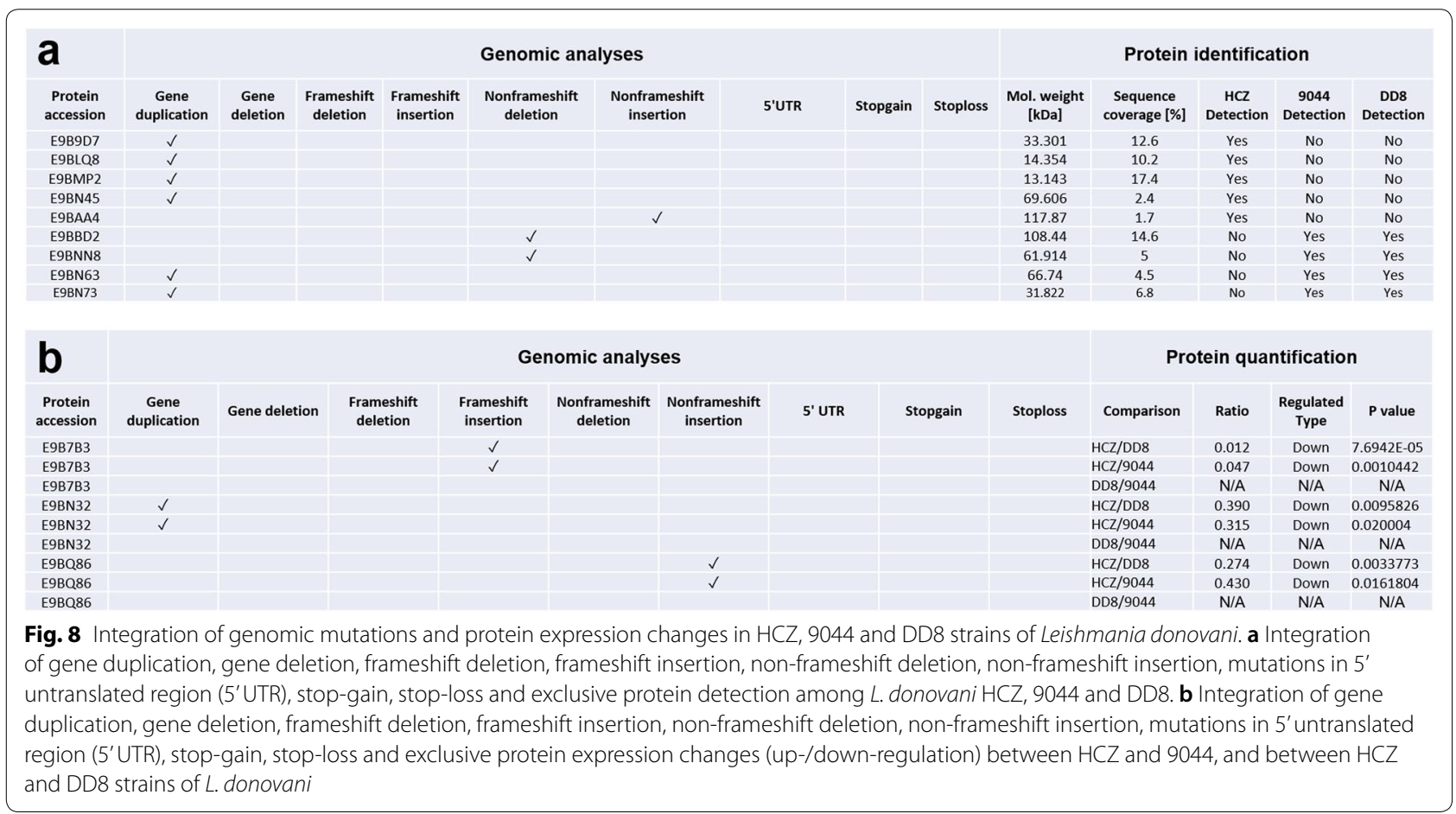

insertion and non-frameshift insertion linked to significant downregulated $(\mathrm{FC} \geq 2$ and $P<0.05)$ abundances of three proteins (E9BN32, E9B7B3 and E9BQ86) in the resistant $\mathrm{HCZ}$ isolate (Fig. 8b, Additional file 1: Table S10). Although most of these molecules were uncharacterised proteins, some of them were inferred to be associated with nucleotide-binding, fatty acid metabolism (biosynthesis and degradation), oxidation-reduction and transport of drugs (Additional file 1: Tables S9 and S10). 


\section{Discussion}

In this study, we confirmed the virulence and antimonyresistance of a previously reported clinical isolate (HCZ) of $L$. donovani [18], compared genomic and proteomic datasets between this drug-resistant isolate and two drug-susceptible strains of $L$. donovani. By excluding natural variations, gene mutations and proteomic variations were identified in the virulent and resistant $\mathrm{HCZ}$ isolate. By integrating the phenotypic, genomic and proteomic results, we identified specific genetic features that might contribute to the pathogenicity and antimony-resistance in HCZ isolate of L. donovani.

Long-term environmental adaptations usually serve as the drivers of genetic mutations in organisms. The development of virulence and resistance in $L$. donovani is essentially environmental selections of genomic variations allowing for fitness gain. Identifying such variations at DNA level would be useful to unravel the mechanisms underlying pathogenicity and drug resistance, which can be achieved nowadays by high-throughput next generation sequencing of genomic DNA and comparative analyses between/among strains [43]. For instance, a recent longitudinal genome analyses of 10 clinical isolates of Leishmania species have shown that gain and loss of gene copies might be the drivers of strain-specific phenotypic variations [27]. Particularly, sequencing the resistant and susceptible strains of Leishmania species has revealed a number of mutations (SNPs and CNVs) that might play roles in the resistance of the parasite [26, 28]. Similarly, in the present study, hundreds of CNVs were identified in the HCZ, DD8 and 9044 strains of $L$. donovani, compared with the reference BPK282A1 strain. However, the considerable overlaps between the resistant (HCZ) isolate and susceptible (DD8 and 9044) strains significantly hindered the interpretation of these findings. Nonetheless, by excluding natural variations indicated between DD8 and 9044 strains, variations specifically identified in the resistant isolate $(n=86)$ are more likely involved in the establishment of resistance and/or virulence. However, linking these genomic variations/mutations to pathogenic or resistant phenotypes is still difficult. The difficulty results from the presence of frequent aneuploidy (the condition of having an abnormal number of chromosomes in a haploid set) in Leishmania spp., which means adequate read depth is needed to ensure the accuracy of detected genetic variations, such as the loss and gain of gene copies [41]. In addition, limited information at post-genomic (RNA, protein and metabolite) levels also challenges the biological exploration and understanding of the genetic variations [44].

A proteomic approach was elected to address these issues in the present study. Although RNA sequencing and transcriptomic analysis have been extensively used to explore molecular alterations that might be associated with drug resistance in Leishmania species [29], such methodology was not employed in this work. This is because post-transcriptional regulations (via microRNAs and other non-coding small RNAs) appear to substantially change the genetic information processing from messenger RNA to protein $[45,46]$. Information captured at protein expression level might be more reliable to reflect the biological differences between resistant and susceptible strains of the parasite [47]. Indeed, proteomic studies of Leishmania spp. have contributed significantly to understanding the surval, infection and drug resistance of this and related parasite [30, 47]. For instance, by comparing the protein abundance between stains of different phenotypes, molecules such as alpha-tubulin, cyclophilin-A, heat-shock proteins, pteridine reductase, superoxide dismutase and tryparedoxin peroxidase have been linked to the resistance and/or virulence of Leishmania species [48, 49]. In this study, significant and specific expression changes were identified for 82 proteins between the resistant and susceptible strains of $L$. donovani. Particularly, the exclusively detected $(n=$ $69)$ and non-detected $(n=66)$ proteins in the resistant (HCZ) isolate of $L$. donovani are very likely to be associated with the establishment of drug resistance. However, although increased tolerance of $L$. donovani to antileishmanial drugs has been linked to reduced drug accumulation, increased infectivity and resistance to oxidative stress [50], little is known about the connections between genetic factors and molecular alterations driving such biological processes.

Integrating the genetic variations revealed by comparative genomics and the molecular changes indicated by comparative proteomics might be useful to answer the biological questions pertaining to the virulence and drug resistance in Leishmania species. Specifically, a recent work integrated proteomic and metabolomic datasets, which sufficiently revealed that changes in glycoconjugate production and redox homeostasis might result in reduced virulence of Leishmania parasite [31]. Therefore, integrating multiple omics datasets might be an efficient way to link biochemistry and molecular biology to phenotypes (e.g. virulence and resistance) [51]. In the present study, although thousands of mutations/variations were identified in protein-coding genes, and substantial changes were indicated in protein abundances, by indepth integration only 12 mutations at DNA level were directly linked to protein expression alterations in the resistant clinical isolate of $L$. donovani (Fig. 8). Although the functional network of these 12 genes/proteins is not clear, they are very likely play roles in the pathogenesis 
and establishment of resistance in host, which might be achieved by altering mRNA transcription and cellular signalling. Nonetheless, although insertion and point mutations of $\mathrm{H} 3$ coding gene were detected in $\mathrm{HCZ}$ isolate, no significant protein expression change of this gene was identified between the resistant isolate and sensitive strains. Clearly, further detailed and integrative studies should be conducted to elucidate such aspects between resistant and susceptible strains of Leishmania spp., preferably at DNA, RNA, protein and metabolites levels. Since it is possible to perform high-throughput CRISPRCas9 mediated genome editing in L. donovani [52-54], further functional studies of these genes and related gene networks would be also useful for more detailed information about the biological alterations allowing for the drug tolerance. Although the present study focused on the promastigote stage of resistant and susceptible strains of $L$. donovani, the findings are expected in the amastigote stage as well, since similar resistant phenotype was identified in the amastigotes of the parasite. Further explorations on the latter stage would provide more comprehensive information about virulence within host animal and tolerance to drugs in vivo.

\section{Conclusions}

In conclusion, high-level pathogenicity and evident antimony-resistance were confirmed for the $\mathrm{HCZ}$ isolate of $L$. donovani. Integrative genomic, proteomic and phenotypic studies of the resistant $\mathrm{HCZ}$ isolate and reference sensitive strains (DD8 and 9044) of $L$. donovani revealed genetic variations likely associated with nucleotidebinding, fatty acid metabolism, oxidation-reduction and transport of drugs. Further functional studies of such aspects would provide novel insights into our understanding of the mechanisms underlying pathogenesis and drug resistance in $L$. donovani and related species.

\section{Supplementary information}

Supplementary information accompanies this paper at https://doi. org/10.1186/s13071-020-04397-4.

\footnotetext{
Additional file 1: Table S1. Statistical summary of genomic analyses of Leishmania donovani strains. Table S2. Exclusive copy number variation identified in Leishmania donovani HCZ strain. Table S3. Exclusive frameshift mutations identified in Leishmania donovani HCZ strain. Table S4. Exclusive nonsense mutations identified in Leishmania donovani HCZ strain. Table S5. Exclusive genomic mutations identified in untranslated region and non-coding RNAs of Leishmania donovani HCZ strain. Table S6. Statistical summary of proteomic analyses of Leishmania donovani strains. Table S7. Protein identification in Leishmania donovani HCZ, DD8 and 9044 strains. Table S8. Differential analysis of protein expression among Leishmania donovani HCZ, DD8 and 9044 strains. Table S9. Integrative analysis of genetic mutation and protein identification in Leishmania donovani HCZ strain. Table S10. Integrative analysis of genetic mutation and protein quantification in Leishmania donovani HCZ strain.
}

\section{Abbreviations}

VL: visceral leishmaniasis; SSG: sodium stibogluconate; PAT: potassium antimonyl tartrate trihydrate; FBS: fetal bovine serum; PBS: phosphate-buffered saline; LDU: Leishman-Donovan Units; FITC: fluorescein isothiocyanate; SV: structural variation; CNV: copy number variation; SNP: single nucleotide polymorphism; LC-MS/MS: liquid chromatographytandem mass spectrometry; FDR: false discovery rate; GO: gene ontology; KEGG: Kyoto encyclopedia of genes and genomes; PCA: principal components analysis; HCA: hierarchical cluster analysis; SD: standard deviation.

\section{Acknowledgements}

The authors are very grateful to Professor Junyun Wang from the National Institute of Parasitic Diseases, Chinese Center for Disease Control and Prevention, Shanghai, China, for providing L. donovani strains MHOM/CN/IN/80/DD8 and $\mathrm{MHOH} / \mathrm{CN} / 90 / 9044$.

\section{Authors' contributions}

$Z W Z$, JL, and JPC conceived and designed the experiments. ZWZ and GXM collected and analysed the data. JL and ZWZ wrote the paper. GXM and ARS revised the paper and polished the language. All authors read and approved the final manuscript.

\section{Funding}

This study was supported by the National Natural Science Foundation of China to JL (31802184) and JC (81672048).

\section{Availability of data and materials}

The datasets supporting the conclusions of this article are included within the article. Genome sequencing data sets have been submitted to the National Center for Biotechnology Information (NCBI) sequence reads archive (SRA), which can be accessed under accession number PRJNA600762. Proteomic data sets have been submitted to the ProteomeXchange Consortium via the PRIDE partner repository under the accession number PXD017089.

\section{Ethics approval and consent to participate}

The use of animals in this study was approved by Sichuan University Medical Ethics Committee (permit no. K2018056). All animal maintenance and experiments were conducted in facilities of Department of Pathogenic Biology, West China School of Basic Medical Sciences and Forensic Medicine, Sichuan University, China, in accordance with national and institutional ethical rules for the care and use of animals.

\section{Consent for publication}

Not applicable.

\section{Competing interests}

The authors declare that they have no competing interests.

\section{Author details}

${ }^{1}$ Department of Pathogenic Biology, West China School of Basic Medical Sciences and Forensic Medicine, Sichuan University, Chengdu, China. ${ }^{2}$ Animal Disease Prevention and Food Safety Key Laboratory of Sichuan Province, Chengdu, China. ${ }^{3}$ College of Animal Sciences, Zhejiang Provincial Key Laboratory of Preventive Veterinary Medicine, Zhejiang University, Hangzhou, China. ${ }^{4}$ Department of Veterinary Biosciences, Melbourne Veterinary School, The University of Melbourne, Parkville, Victoria, Australia. ${ }^{5}$ Department of Pathology, Ohio State University Medical Center, Ohio State University, Columbus, USA.

${ }^{6}$ Department of Microbiology, Ohio State University, Columbus, USA.

Received: 14 July 2020 Accepted: 7 October 2020

Published online: 12 October 2020

\section{References}

1. Karimkhani C, Wanga V, Coffeng LE, Naghavi P, Dellavalle RP, Naghavi M. Global burden of cutaneous leishmaniasis: a cross-sectional analysis from the global burden of disease study 2013. Lancet Infect Dis. 2016;16:584-91. 
2. Burza S, Croft SL, Boelaert M. Leishmaniasis. Lancet. 2018;392:951-70.

3. Herwaldt BL. Leishmaniasis. Lancet. 1999;354:1191-9.

4. Murray HW, Berman JD, Davies CR, Saravia NG. Advances in leishmaniasis. Lancet. 2005;366:1561-77.

5. Abeijon C, Campos-Neto A. Potential non-invasive urine-based antigen (protein) detection assay to diagnose active visceral leishmaniasis. PLoS Negl Trop Dis. 2013;7:e2161.

6. Bekhit AA, El-Agroudy E, Helmy A, Ibrahim TM, Shavandi A, Bekhit AE. Leishmania treatment and prevention: natural and synthesized drugs. Eur J Med Chem. 2018;160:229-44.

7. Dixit KK, Verma S, Singh OP, Singh D, Singh AP, Gupta R, et al. Validation of SYBR green I based closed tube loop mediated isothermal amplification (LAMP) assay and simplified direct-blood-lysis (DBL)-LAMP assay for diagnosis of visceral leishmaniasis (VL). PLoS Negl Trop Dis. 2018;12:e0006922.

8. Lun Z-R, Wu M-S, Chen Y-F, Wang J-Y, Zhou X-N, Liao L-F, et al. Visceral leishmaniasis in China: an endemic disease under control. Clin Microbiol Rev. 2015;28:987-1004.

9. Fiuza JA, Gannavaram S, Santiago HDC, Selvapandiyan A, Souza DM, Passos LSA, et al. Vaccination using live attenuated Leishmania donovani centrin deleted parasites induces protection in dogs against Leishmania infantum. Vaccine. 2015;33:280-8.

10. Duthie MS, Pereira L, Favila M, Hofmeyer KA, Reed SJ, Metangmo S, et al. A defined subunit vaccine that protects against vector-borne visceral leishmaniasis. NPJ Vaccines. 2017;2:23.

11. Osman M, Mistry A, Keding A, Gabe R, Cook E, Forrester S, et al. A third generation vaccine for human visceral leishmaniasis and post kala azar dermal leishmaniasis: first-in human trial of ChAd63-KH. PLoS Negl Trop Dis. 2017;11:e0005527.

12. Desjeux P. Therapeutic options for visceral leishmaniasis. Med Mal Infect. 2005;35(Suppl. 2):S74-6.

13. Monge-Maillo B, López-Vélez R. Therapeutic options for visceral leishmaniasis. Drugs. 2013;73:1863-88.

14. Guerin PJ, Olliaro P, Sundar S, Boelaert M, Croft SL, Desjeux P, et al. Visceral leishmaniasis: current status of control, diagnosis, and treatment, and a proposed research and development agenda. Lancet Infect Dis. 2002;2:494-501

15. Ostyn B, Uranw S, Bhattarai NR, Das ML, Rai K, Tersago K, et al. Transmission of Leishmania donovani in the hills of eastern Nepal, an outbreak investigation in Okhaldhunga and Bhojpur districts. PLoS Negl Trop Dis. 2015;9:e0003966.

16. Ponte-Sucre A, Gamarro F, Dujardin J-C, Barrett MP, López-Vélez R, García Hernández $\mathrm{R}$, et al. Drug resistance and treatment failure in leishmaniasis: a 21st century challenge. PLoS Negl Trop Dis. 2017;11:e0006052.

17. Zijlstra EE, Alves F, Rijal S, Arana B, Alvar J. Post-kala-azar dermal leishmaniasis in the Indian subcontinent: a threat to the South-east Asia region Kala-azar elimination programme. PLoS NegI Trop Dis. 2017;1 1:e0005877.

18. He J, Huang F, Zhang J, Chen $\mathrm{H}$, Chen Q, Zhang J, et al. DNA primeprotein boost vaccine encoding HLA-A2, HLA-A24 and HLA-DR1 restricted epitopes of CaNA2 against visceral leishmaniasis. Immunology. 2019;156:94-108.

19. Hendrickx S, Guerin PJ, Caljon G, Croft SL, Maes L. Evaluating drug resistance in visceral leishmaniasis: the challenges. Parasitology. 2018:145:453-63.

20. Dujardin J-C. Structure, dynamics and function of Leishmania genome: resolving the puzzle of infection, genetics and evolution? Infect Genet Evol. 2009;9:290-7.

21. Cantacessi C, Dantas-Torres F, Nolan MJ, Otranto D. The past, present, and future of Leishmania genomics and transcriptomics. Trends Parasitol. 2015;31:100-8.

22. Cruz AK, Freitas-Castro F. Genome and transcriptome analyses of Leishmania spp: opening Pandora’s box. Curr Opin Microbiol. 2019;52:64-9.

23. Wincker P, Ravel C, Blaineau C, Pages M, Jauffret Y, Dedet JP, et al. The Leishmania genome comprises 36 chromosomes conserved across widely divergent human pathogenic species. Nucleic Acids Res. 1996:24:1688-94.

24. Ivens AC, Peacock CS, Worthey EA, Murphy L, Aggarwal G, Berriman M, et al. The genome of the kinetoplastid parasite, Leishmania major. Science. 2005;309:436-42.
25. Peacock CS, Seeger K, Harris D, Murphy L, Ruiz JC, Quail MA, et al. Comparative genomic analysis of three Leishmania species that cause diverse human disease. Nat Genet. 2007:39:839-47.

26. Downing T, Imamura H, Decuypere S, Clark TG, Coombs GH, Cotton JA, et al. Whole genome sequencing of multiple Leishmania donovani clinical isolates provides insights into population structure and mechanisms of drug resistance. Genome Res. 2011;21:2143-56.

27. Bussotti G, Gouzelou E, Côrtes Boité M, Kherachi I, Harrat Z, Eddaikra N, et al. Leishmania genome dynamics during environmental adaptation reveal strain-specific differences in gene copy number variation, karyotype instability, and telomeric amplification. MBio. 2018;9:e01399.

28. Carnielli JBT, Crouch K, Forrester S, Silva VC, Carvalho SFG, Damasceno JD, et al. A Leishmania infantum genetic marker associated with miltefosine treatment failure for visceral leishmaniasis. EBioMedicine. 2018;36:83-91.

29. Rastrojo A, García-Hernández R, Vargas P, Camacho E, Corvo L, Imamura $\mathrm{H}$, et al. Genomic and transcriptomic alterations in Leishmania donovani lines experimentally resistant to antileishmanial drugs. Int J Parasitol Drugs Drug Resist. 2018:8:246-64.

30. Paape D, Aebischer T. Contribution of proteomics of Leishmania spp to the understanding of differentiation, drug resistance mechanisms, vaccine and drug development. J Proteomics. 2011;74:1614-24.

31. Akpunarlieva S, Weidt S, Lamasudin D, Naula C, Henderson D, Barrett M, et al. Integration of proteomics and metabolomics to elucidate metabolic adaptation in Leishmania. J Proteomics. 2017;155:85-98.

32. Douanne N, Wagner V, Roy G, Leprohon P, Ouellette M, Fernandez-Prada C. MRPA independent mechanisms of antimony resistance in Leishmania infantum. Int J Parasitol Drugs Drug Resist. 2020;13:28-37.

33. Zheng Zhiwan, Li Jiao, Chen Han, He Jinlei, Chen Qiwei, Zhang Jianhui, et al. Evaluation of in vitro antileishmanial efficacy of cyclosporin A and its non-immunosuppressive derivative, dihydrocyclosporin A. Parasit Vectors. 2020;13:94.

34. Varikuti S, Volpedo G, Saljoughian N, Hamza OM, Halsey G, Ryan NM, et al. The potent ITK/BTK inhibitor ibrutinib is effective for the treatment of experimental visceral leishmaniasis caused by Leishmania donovani. J Infect Dis. 2019;219:599-608.

35. Li H, Durbin R. Fast and accurate long-read alignment with BurrowsWheeler transform. Bioinformatics. 2010;26:589-95.

36. Chen K, Wallis JW, McLellan MD, Larson DE, Kalicki JM, Pohl CS, et al. BreakDancer: an algorithm for high-resolution mapping of genomic structural variation. Nat Methods. 2009;6:677-81.

37. Abyzov A, Urban AE, Snyder M, Gerstein M. CNVnator: an approach to discover, genotype, and characterize typical and atypical CNVs from family and population genome sequencing. Genome Res. 2011;21:974-84.

38. McKenna A, Hanna M, Banks E, Sivachenko A, Cibulskis K, Kernytsky A, et al. The genome analysis toolkit: a mapreduce framework for analyzing next-generation DNA sequencing data. Genome Res. 2010;20:1297-303.

39. Wang $\mathrm{K}$, Li M, Hakonarson H. ANNOVAR: functional annotation of genetic variants from high-throughput sequencing data. Nucleic Acids Res. 2010;38:e164

40. Tyanova S, Temu T, Cox J. The MaxQuant computational platform for mass spectrometry-based shotgun proteomics. Nat Protoc. 2016;11:2301-19.

41. Tyanova S, Temu T, Sinitcyn P, Carlson A, Hein MY, Geiger T, et al. The Perseus computational platform for comprehensive analysis of (prote)omics data. Nat Methods. 2016;13:731-40.

42. Tyanova S, Cox J. Perseus: a bioinformatics platform for integrative analysis of proteomics data in cancer research. Methods Mol Biol. 2018;1711:133-48.

43. Imamura H, Dujardin J-C. A guide to next generation sequence analysis of Leishmania Genomes. Methods Mol Biol. 2019;1971:69-94.

44. Berg M, Mannaert A, Vanaerschot M, Van Der Auwera G, Dujardin J-C. (Post-) Genomic approaches to tackle drug resistance in Leishmania. Parasitology. 2013;140:1492-505.

45. Devaney E, Winter AD, Britton C. microRNAs: a role in drug resistance in parasitic nematodes? Trends Parasitol. 2010;26:428-33.

46. Ma G, Wang T, Korhonen PK, Ang C-S, Williamson NA, Young ND, et al. Molecular alterations during larval development of Haemonchus contortus in vitro are under tight post transcriptional control. Int J Parasitol. 2018:48:763-72. 
47. Veras PST, Bezerra de Menezes JP. Using proteomics to understand how Leishmania parasites survive inside the host and establish infection. IJMS. 2016;17:1270.

48. Matrangolo FSV, Liarte DB, Andrade LC, de Melo MF, Andrade JM, Ferreira RF, et al. Comparative proteomic analysis of antimony-resistant and -susceptible Leishmania braziliensis and Leishmania infantum chagasi lines. Mol Biochem Parasitol. 2013;190:63-75.

49. de Rezende E, Kawahara R, Peña MS, Palmisano G, Stolf BS. Quantitative proteomic analysis of amastigotes from Leishmania (L) amazonensis LV79 and PH8 strains reveals molecular traits associated with the virulence phenotype. PLoS Negl Trop Dis. 2017;11:e0006090.

50. Deep DK, Singh R, Bhandari V, Verma A, Sharma V, Wajid S, et al. Increased miltefosine tolerance in clinical isolates of Leishmania donovani is associated with reduced drug accumulation, increased infectivity and resistance to oxidative stress. PLoS Negl Trop Dis. 2017;11:e0005641.

51. Ma G, Wang T, Korhonen PK, Hofmann A, Sternberg PW, Young $N D$, et al. Elucidating the molecular and developmental biology of parasitic nematodes: moving to a multiomics paradigm. Adv Parasitol. 2020;108:175-229.

52. Sollelis L, Ghorbal M, MacPherson CR, Martins RM, Kuk N, Crobu L, et al. First efficient CRISPR-Cas9-mediated genome editing in Leishmania parasites. Cell Microbiol. 2015;17:1405-12.

53. Zhang W-W, Matlashewski G. CRISPR-Cas9-mediated genome editing in Leishmania donovani. MBio. 2015;6:e00861.

54. Beneke T, Madden R, Makin L, Valli J, Sunter J, Gluenz E. A CRISPR Cas9 high throughput genome editing toolkit for kinetoplastids. R Soc Open Sci. 2017:4:170095.

\section{Publisher's Note}

Springer Nature remains neutral with regard to jurisdictional claims in published maps and institutional affiliations.
Ready to submit your research? Choose BMC and benefit from:

- fast, convenient online submission

- thorough peer review by experienced researchers in your field

- rapid publication on acceptance

- support for research data, including large and complex data types

- gold Open Access which fosters wider collaboration and increased citations

- maximum visibility for your research: over $100 \mathrm{M}$ website views per year

At BMC, research is always in progress.

Learn more biomedcentral.com/submissions 